\title{
Principle and analysis of multiturn injection using stable islands of transverse phase space
}

\author{
M. Giovannozzi and J. Morel \\ CERN, CH 1211 Geneva 23, Switzerland \\ (Received 2 February 2007; published 9 March 2007)
}

\begin{abstract}
By applying a time reversal to the multiturn extraction method recently proposed, a novel approach to perform multiturn injection is described. It is based on the use of stable islands of the horizontal phase space generated by means of sextupoles and octupoles. A particle beam can be injected into stable islands of phase space, and then a slow tune variation allows merging the beam trapped inside the islands. The results of numerical simulations will be presented and discussed in detail, showing how to use the proposed approach to generate hollow bunches in the transverse phase space, and how different resonances can be used. The dependence of the final beam parameters on the key quantities of the proposed approach, such as emittance of the initial beam and the way the resonance is crossed, is investigated. Furthermore, a comparison of the proposed approach against the classical multiturn injection without the use of stable islands is carried out.
\end{abstract}

DOI: 10.1103/PhysRevSTAB.10.034001

PACS numbers: 29.27.Ac, 29.27.Ac, 29.27.Bd, 05.45.-a

\section{INTRODUCTION}

The injection of charged particles into a circular accelerator can be performed by three distinct approaches: single-turn, multiturn, and charge-exchange injection [1]. The first method is based on the use of a septum magnet to deflect the injected beam towards the central orbit of the receiving machine and a fast deflector, a so-called kicker, to adjust the angle of the incoming beam so to match both position and angle of the central orbit. While the septum magnet can be pulsed or not, the kicker provides a deflection over one machine circumference only, which will be the length of the injected pulse. The second method aims at injecting more than one turn. This is normally obtained by means of a septum magnet and an appropriate timedependent bump used to paint the phase space so to generate the appropriate transverse and/or longitudinal beam distribution. Finally, the charge-exchange method [2] is a refined version of the multiturn injection, where $\mathrm{H}^{-}$ions are injected and stripped at the injection point: this has the advantage of generating high-brightness beams.

In recent years, a novel method to perform multiturn extraction from a circular particle accelerator was proposed [3-6]. It is based on particle trapping inside stable islands of transverse phase space generated by nonlinear magnetic fields, such as sextupolar or octupolar ones. This method proved to work well not only in numerical simulations [3-6], but also in a series of experiments carried out at the CERN Proton Synchrotron (PS) machine [7-10]. It is worth mentioning that the novel method for performing multiturn extraction will be implemented in the PS machine as a replacement of the current continuous transfer extraction mode (see Ref. [11] for more details in the implementation project).
The time-reversal property of the dynamics involved in the novel extraction process allows extending the approach to perform multiturn injection. The idea consists in injecting the beam in stable islands of phase space and then to vary the tune so to merge back the beamlets into one single beam $[12,13]$. This requires a closed orbit bump generated by kickers so that one of the stable islands is used to inject the beam. Then, once all the islands are filled, the bump is collapsed and the tune is varied to change the beam distribution towards the final shape. The number of injected turns depends on the resonance used for generating the stable islands. In the next sections, the results of numerical simulations performed on a simple model are presented to support the validity of the proposed concept.

Parenthetically, it is worth noting that a proposal of injecting the beam into stable islands of the transverse phase space generated by an octupolar resonance was already made in Ref. [14] a long time ago. However, to our best knowledge, such a proposal never led to more detailed and quantitative theoretical studies and experimental tests. The results reported in this paper represent an independent and complete analysis of a novel injection process originated from the studies for a novel multiturn extraction.

The plan of the paper is the following: in Sec. II the results of numerical simulations for the proposed technique are presented and discussed in detail for the fourth order, in Sec. II B, and for the third-order resonance, in Sec. II C. The analysis of how the shape of the final beam distribution depends on the details of the injection process is considered in Sec. II D. In Sec. III a simple model of the classical multiturn injection is presented, which is then used to compare the performance of both approaches. Finally, some conclusions are drawn in Sec. IV. The details of the analytical computations to determine the final beam pa- 
rameters of the classical multiturn injection are derived in Appendix A.

\section{NUMERICAL SIMULATIONS}

\section{A. The model}

The model used to study the proposed injection technique consists of a simple focusing/defocusing (FODO) cell with a sextupole and an octupole magnet located at the same longitudinal position, both represented in the singlekick approximation [15]. For the application under study, only the horizontal plane is relevant. Therefore, the evolution of the beam dynamics can be obtained by using a $2 \mathrm{D}$ one-turn polynomial transfer map of the form

$$
\left(\begin{array}{c}
\hat{X} \\
\hat{X}^{\prime}
\end{array}\right)_{n+1}=R[\omega(n)]\left(\begin{array}{c}
\hat{X} \\
\hat{X}^{\prime}+\hat{X}^{2}+\kappa \hat{X}^{3}
\end{array}\right)_{n} .
$$

The components of the vector $\left(\hat{X}, \hat{X}^{\prime}\right)$ are dimensionless coordinates allowing to set the coefficient of the quadratic term of the map (1) to one [15]. $R[\omega(n)]$ represents a rotation matrix of an angle $\omega(n)=2 \pi \nu(n)$ and $\kappa$ depends on the ratio between the strength of the sextupole and the octupole weighted by the value of the optical beta-function at the location of the nonlinear magnetic elements [6], namely,

$$
\kappa=\frac{2}{3} \frac{K_{3}}{K_{2}^{2}} \frac{1}{\beta}
$$

In terms of the usual development of the magnetic field into multipoles up to order $m$, one has that

$$
\begin{aligned}
B_{y}(x, y ; s)+i B_{x}(x, y ; s)= & B_{0}\left\{\rho_{0} \sum_{n=1}^{m}\left[K_{n}(s)+i J_{n}(s)\right]\right. \\
& \left.\times \frac{(x+i y)^{n}}{n !}-\chi(s)\right\}
\end{aligned}
$$

where $\chi(s)$ is a function equal to 1 in dipoles and 0 elsewhere. The coefficients $K_{n}, J_{n}$, respectively, the normal and skew normalized integrated multipole strengths, are defined according to

$$
K_{n}=\frac{1}{B_{0} \rho} \frac{\partial^{n} B_{y}}{\partial x^{n}} \ell \quad J_{n}=\frac{1}{B_{0} \rho} \frac{\partial^{n} B_{x}}{\partial x^{n}} \ell .
$$

In Eqs. (2) and (3), $B_{0} \rho$ stands for the magnetic rigidity of the reference particle, $B_{x}, B_{y}$ are the transverse components of the magnetic field, and $\ell$ is the physical length of the magnetic element. In the following it will be assumed that $J_{n}=0$ for $n \geq 1$.

The relation between the physical coordinates $\left(x, x^{\prime}\right)$ and the standard Courant-Snyder ones $\left(\hat{x}, \hat{x}^{\prime}\right)$ is given in Ref. [16] and also reported in Eq. (7), while the adimensional normalized coordinates $\left(\hat{X}, \hat{X}^{\prime}\right)$ are derived according to

$$
\left(\hat{x}, \hat{x}^{\prime}\right)=\lambda\left(\hat{X}, \hat{X}^{\prime}\right) \quad \text { where } \lambda=\frac{1}{2} K_{2} \beta^{3 / 2}
$$

The angle $\omega(n)$ is indeed a function of the turn number and, for the results presented in Secs. II B and IIC the time dependence of $\omega(n)$ is always chosen linear. The general expression of the tune variation is expressed as

$$
\nu(n)= \begin{cases}\nu_{a}-\left(\nu_{a}-\nu_{b}\right)\left(\frac{n-1}{n_{1}-1}\right)^{p} & \text { if } n \leq n_{1} \\ \nu_{b} & \text { if } n_{1}<n \leq n_{2} \\ \nu_{b}-\left(\nu_{b}-\nu_{c}\right)\left(\frac{n-n_{2}}{n_{3}-n_{2}}\right) & \text { if } n_{2}<n \leq n_{3},\end{cases}
$$

where $\nu_{a}, \nu_{b}, \nu_{c}$ are the initial, intermediate, and final tune values, respectively, $n_{1}, n_{2}, n_{3}$ represent the turn number of transition between the stage of resonance crossing, which occurs in the interval $\left(\nu_{a}, \nu_{b}\right)$, and constant tune, constant tune, and linear ramp to merge islands after injection, respectively. Finally, $p$ is a parameter used to change the functional dependence of the tune variation on $n$ during the resonance crossing. It allows changing from a linear sweep to a power law, thus changing the speed of resonance crossing.

It is worth stressing that in the numerical and analytical computations reported here the special adimensional normalized coordinates are used.

\section{B. Results of simulations: Fourth-order resonance}

The numerical simulations focused on two resonances, namely, the fourth and the third order. In the first case, the injection occurs over four turns. The value of the coefficient $\kappa$ is kept constant throughout the process and equal to -1.5 . The initial beam distribution is chosen to be Gaussian in both position and angle with a sigma of 0.02 in both dimensions. The number of initial conditions is $10^{6}$ equally shared among the four injected islands. The resonance is crossed in $2 \times 10^{4}$ turns and the tune variation is defined by $\left(\nu_{a}, \nu_{b}, \nu_{c}\right)=(0.245,0.250,0.255)$, $\left(n_{1}, n_{2}, n_{3}\right)=(13000,500,6500)$ and $p=1$. The results concerning the evolution of the beam distribution are shown in Fig. 1. The beam is injected using the outermost island lying along the positive horizontal axis, which is supposed to be located beyond the blade of the injection septum. At the end of the first four turns, the injection process proper is over. The beam ellipses are distorted due to the nonlinear effects. The next stage consists in changing the tune so to sweep through the resonance and then to merge the four beamlets into one single structure. The final stage of the beam evolution is rather striking as the beam distribution is not at all Gaussian, but it is a hollow distribution, with the beam spread over an annulus in phase space.

Such an effect is due to the fact that the islands' size is also changing during the whole process, being a function of the linear tune [17]. In particular, the size tends to zero while approaching the exact resonance condition. This means that, whenever the tune is sufficiently near to the resonant value, the islands become so small that almost no beam can be transported towards the origin. 

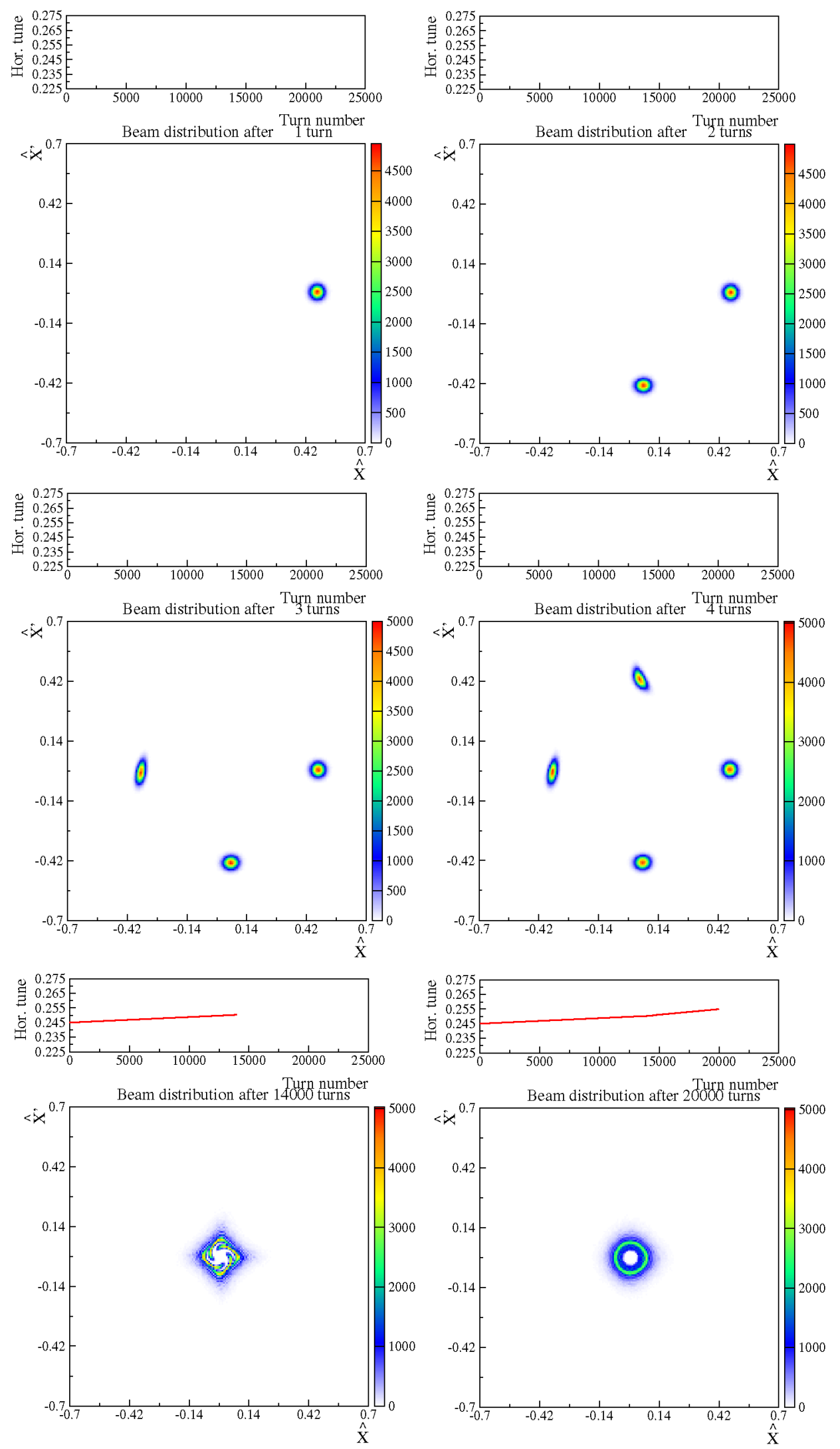

FIG. 1. (Color) Multiturn injection by means of trapping in stable islands of transverse phase space. Four turns are injected and the beamlets merged by crossing the fourth-order resonance. The tune variation is reported in the upper part of each plot. As a result a hollow beam in the transverse phase space is generated. 

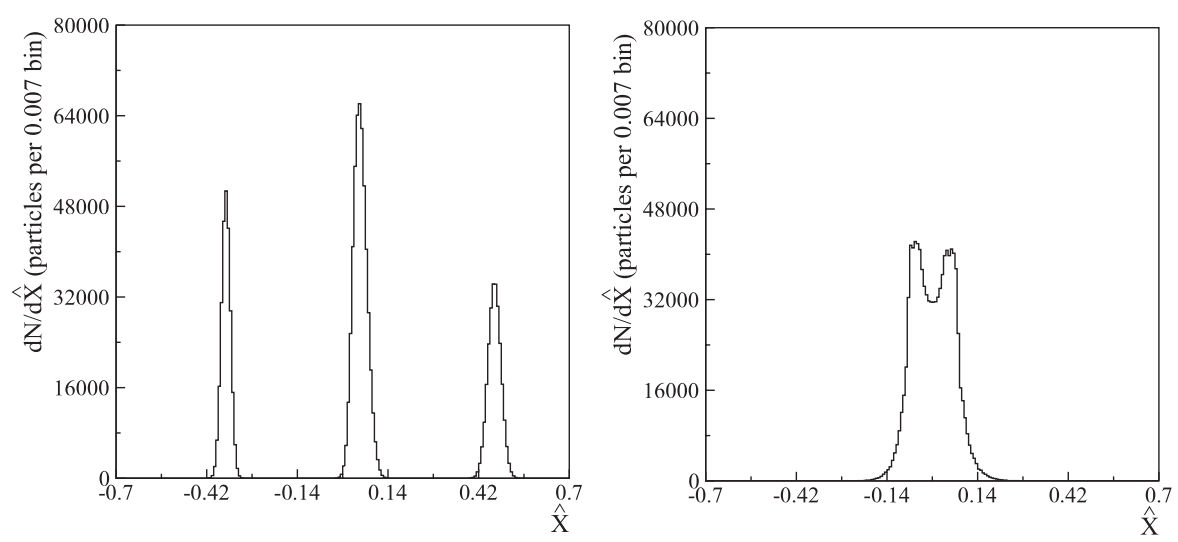

FIG. 2. Projected distribution functions for the four-turn injection right after the injection (left) and at the end of the merging process (right). The two islands centered at the origin with opposite angles are projected onto the central peak visible in the initial distribution function.

The projected beam distribution along the horizontal phase space axis is shown in Fig. 2, where the beamlets just after injection (left part) as well as the final beam (right part) are shown. The three peaks visible in the left part are indeed the result of the projection of the four injected turns shown in Fig. 1 (center right part). Therefore, the single central peak is indeed the superposition of two injected turns. The different width of the three distributions is an effect of the distortion of the beam ellipse induced by the nonlinear effects. The hollow beam distribution shown in the right part of Fig. 2 reflects the observation made that the final beam is not at all Gaussian.

A possible solution to this issue is rather straightforward. As the fourth-order resonance is stable an additional
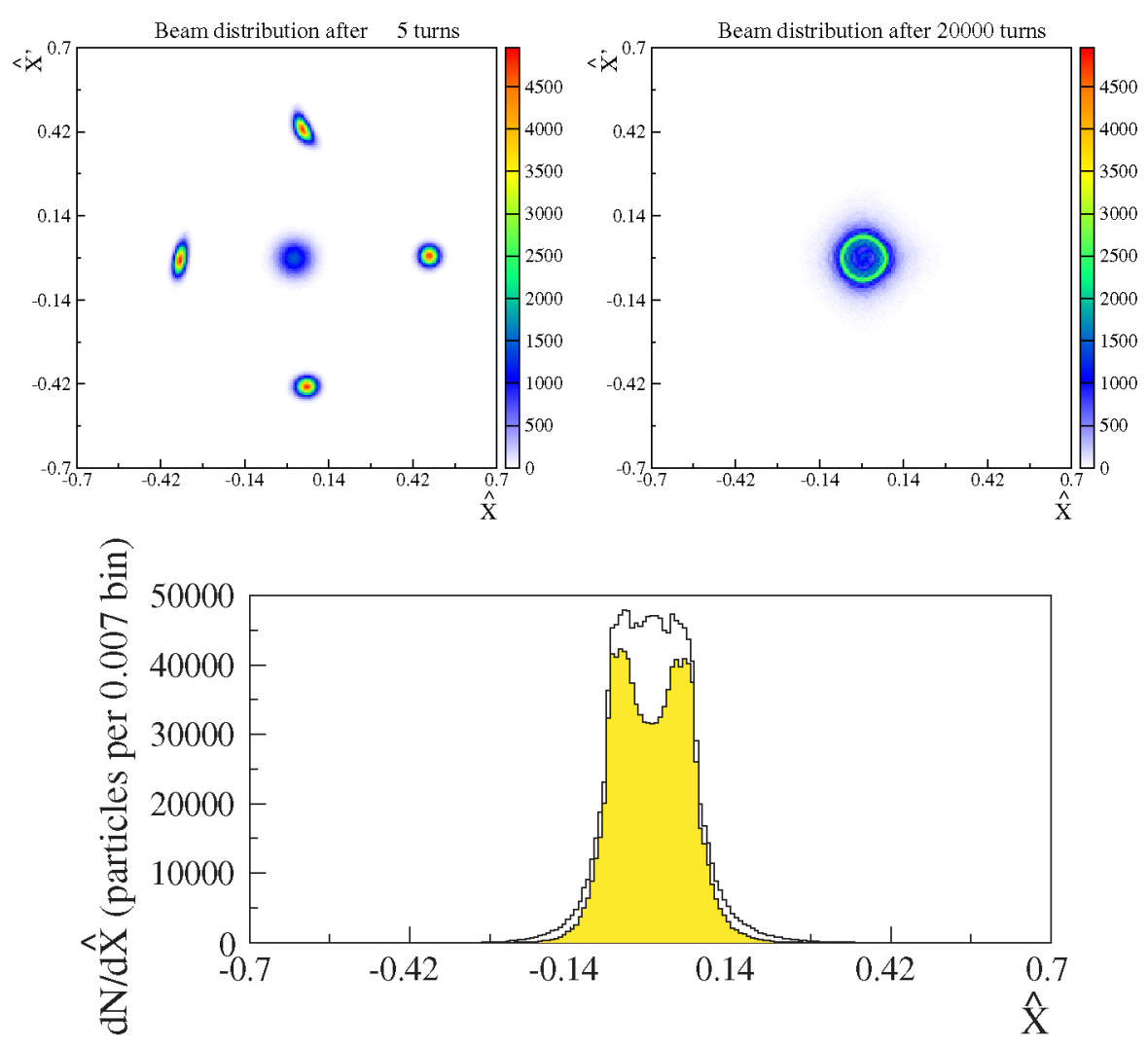

FIG. 3. (Color) Final distribution function for a variant of the proposed four-turn injection with a fifth injected turn so to generate an almost uniform distribution. The beam distribution after the end of the injection process is shown on the upper left part, while the final distribution is reported on the upper right part. The projected beam distribution is shown in the lower part: the final profile for the configuration plotted in Fig. 2 is also shown for comparison (shaded area). 
TABLE I. Parameters of the beam distributions of the five injected turns used to generate the final beam distributions shown in Fig. 3. The offset is expressed in the adimensional coordinates and it is referred to the nominal position of the fixed point at the center of the island.

\begin{tabular}{lcccccc}
\hline \hline & \multicolumn{2}{c}{ Hollow distribution } & \multicolumn{3}{c}{ Quasiuniform distribution } \\
& $\begin{array}{c}\text { Number of } \\
\text { particles }\end{array}$ & $\begin{array}{c}\text { Offset } \\
\left(\Delta \hat{X}, \Delta \hat{X}^{\prime}\right)\end{array}$ & $\sigma$ & pumber of & Offset & \\
\hline First turn & $\ldots$ & $\ldots$ & $\ldots$ & $2.704 \times 10^{5}$ & $(0.02,0.00)$ & 0.038 \\
Last four turns & $2.5 \times 10^{5}$ & $(0,0)$ & 0.02 & $2.5 \times 10^{5}$ & $(0.00,0.00)$ & 0.020 \\
\hline \hline
\end{tabular}

turn could be injected first at the origin of phase space. In practice this implies that the origin of phase space is displaced by the closed bump created by kickers beyond the position of the septum blade. Once the first turn is injected, the bump amplitude is reduced so that the stable island is beyond the septum blade, but not the central orbit: in this condition the injection process can continue for four additional turns. The properties of the first injected turn, i.e., number of particles and sigma, can be used to paint the shape of the final beam distribution, e.g., to fill the hole in the center. Such a feature of the final beam distribution might even turn out to be an interesting point in some conditions, such as when dealing with high-intensity beams. In fact, it is well known that the largest spacecharge tune shift affects particles in the beam core. This explains why efforts are devoted to reducing the beam density around the origin of phase space, in particular, by developing techniques to generate flat or hollow bunches in the longitudinal phase space [18-20]. Therefore, the proposed approach could be used to shape the beam distribution so to reduce the space-charge effects at injection, where they are particularly harmful. As an example, the final beam profile for a possible variant is shown in Fig. 3 together with the one plotted in Fig. 2. The parameters of the tune sweep are the same as those used to generate Fig. 1. The new final beam distribution is obtained by injecting an additional turn at the center of phase space. The parameters, i.e., the number of particles and the sigma, of the injected turns are reported in Table I.

It is important to stress that the simulations presented here do not take into account the Coulomb interaction between the particles, hence the observation made should be confirmed by detailed numerical simulations taking into account also space-charge effects. On the other hand, one could argue that, due to the peculiar beam distribution, space-charge effects should be highly reduced, hence the final beam distribution might be preserved even under the influence of Coulomb interaction.

Finally, it is worthwhile stressing that during the whole injection process no particle's loss is observed.

\section{Results of simulations: Third-order resonance}

A study of the possibility of using the third-order resonance to perform multiturn injection is also carried out.
Even in this case it is possible to complete the injection process without any particle loss. As an example, the beam evolution is shown in Fig. 4. In this case the initial Gaussian distributions feature a sigma of 0.017 and $3 \times 10^{6}$ initial conditions are shared in the three injected distributions while $\kappa=-5$. The parameters for the tune variation are $\left(\nu_{a}, \nu_{b}, \nu_{c}\right)=(0.328,0.340,0.350)$, $\left(n_{1}, n_{2}, n_{3}\right)=(13000,500,6500)$, and $p=1$. The distribution projected along the $\hat{X}$ axis is shown in Fig. 5. The investigation of the possibility of injecting a fourth turn led to different results with respect to the case of the fourthorder resonance. In fact, the third-order resonance is well known to be generically unstable [15]. This implies that the particles injected near the origin will be lost rather quickly. Because of the time dependence of the model used in the numerical simulations, the unstable area around the origin grows in size as long as the tune is moved away from the resonant value. Therefore, no particle is lost, but an extended halo is generated, as one can clearly see in Fig. 6. In this case the distribution injected at the center of the phase space has the same properties of the other three and no offset is applied. This observation leads to the conclusion that injecting a fourth turn around the origin is not a viable option for the proposed multiturn injection based on the use of the third-order resonance.

\section{Dependence of the final beam parameters on injection process}

In the proposed process, the successive injected turns are merged into a single beam by applying an appropriate tune variation after which the final beam distribution is ready to undergo the usual process of, e.g., storage or acceleration. At this stage it is important to avoid any emittance blowup, which might occur in case the final beam distribution is not matched with the nominal optics of the circular machine in which the beam was injected. This might lead to halo generation and, eventually core-emittance blowup and beam losses in case the machine acceptance is reached.

The best way to evaluate the performance of the proposed approach consists of evaluating the betatron mismatch due to the shape of the beam distribution at the end of the injection and merging processes. To quantify the betatron mismatch, it is customary to start from the statistical definition of beam emittance $\epsilon$ and optical parameters 

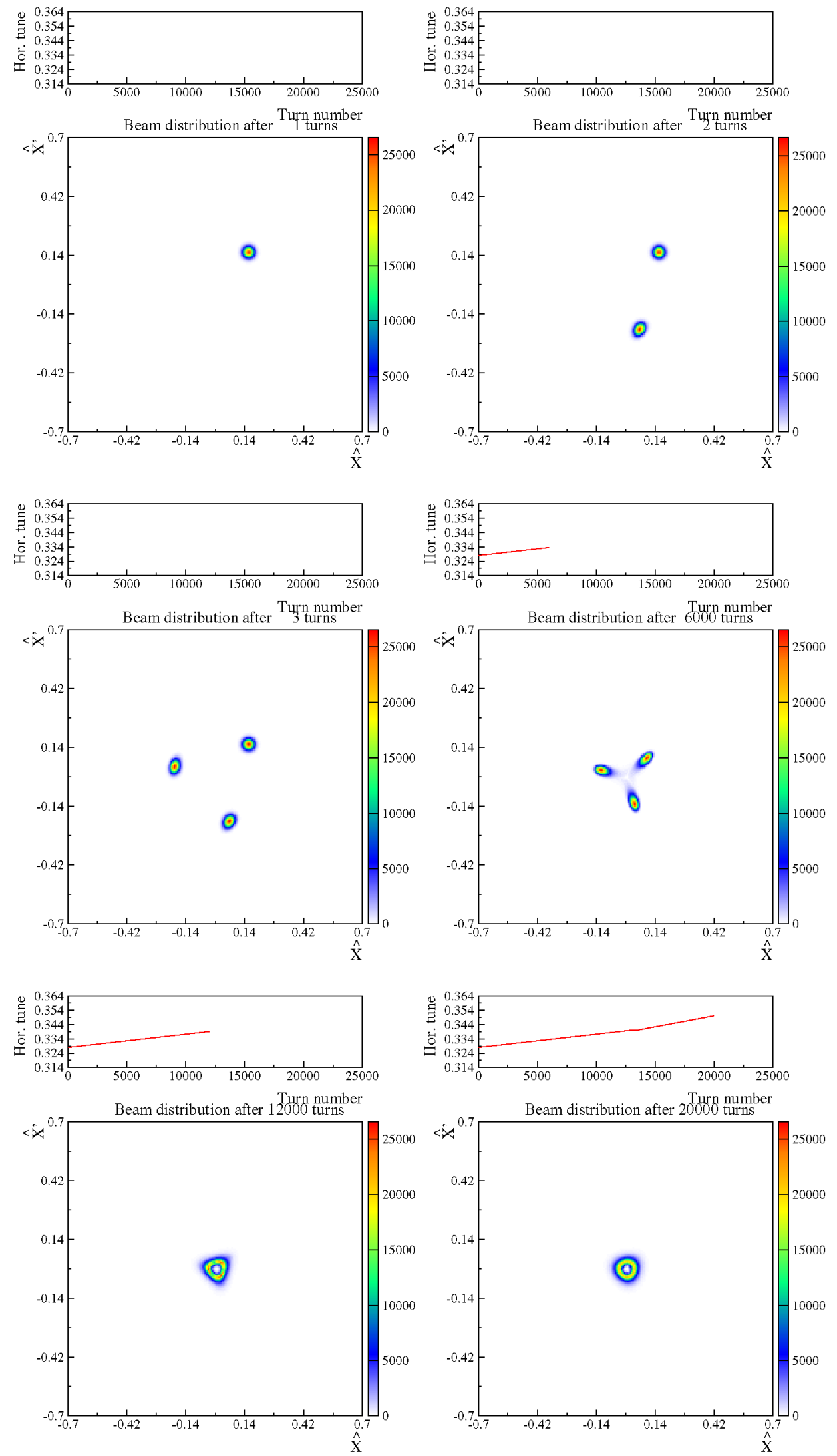

FIG. 4. (Color) Multiturn injection by means of trapping in stable islands of transverse phase space. Three turns are injected and the beamlets merged by crossing the third-order resonance. The tune variation is reported in the upper part of each plot. As for the fourturn injection, a hollow beam in the transverse phase space is generated. 

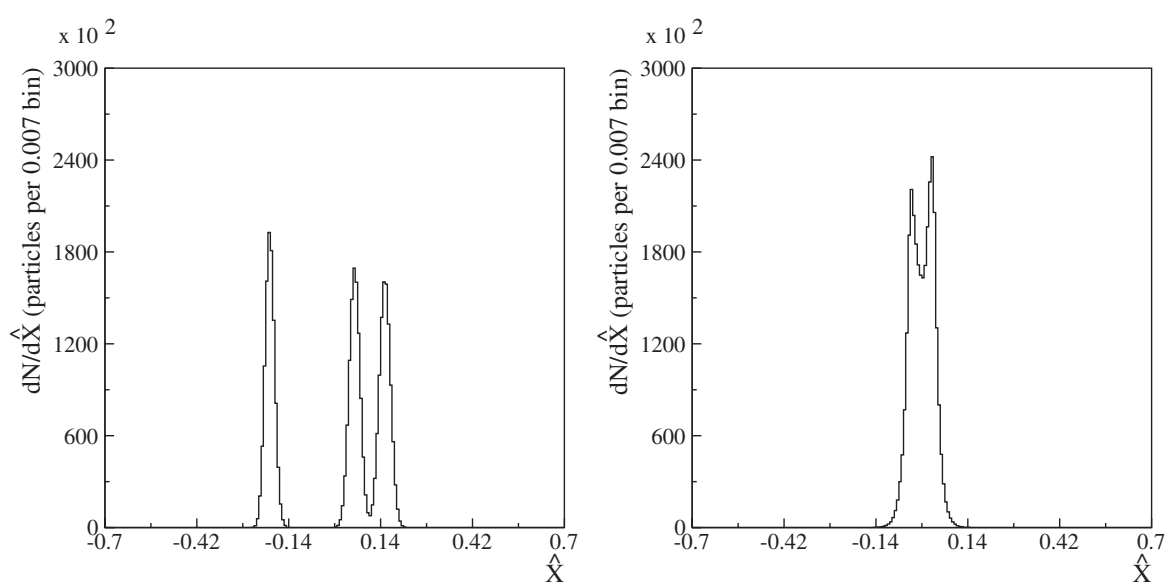

FIG. 5. Projected distribution functions for the three-turn injection after the injection (left) and at the end of the merging process (right). The two islands centered at the origin with opposite angles are projected onto the central peak visible in the initial distribution function.

$\alpha^{*}, \beta^{*}, \gamma^{*}[16]$ in terms of the second-order moments of the beam distribution [21-23]. Using the notations introduced in the previous sections it can be shown that the following holds:

$$
\left\langle x^{2}\right\rangle=\beta^{*} \epsilon \quad\left\langle x x^{\prime}\right\rangle=-\alpha^{*} \epsilon \quad\left\langle x^{\prime 2}\right\rangle=\gamma^{*} \epsilon .
$$

In the equations quoted above, the symbol $\langle\cdot\rangle$ stands for the average over the beam distribution of the specified variable. It is worthwhile mentioning that, whenever the beam distribution is not centered at the origin, the second-order moments in Eqs. (6) are the central ones, i.e., referred to
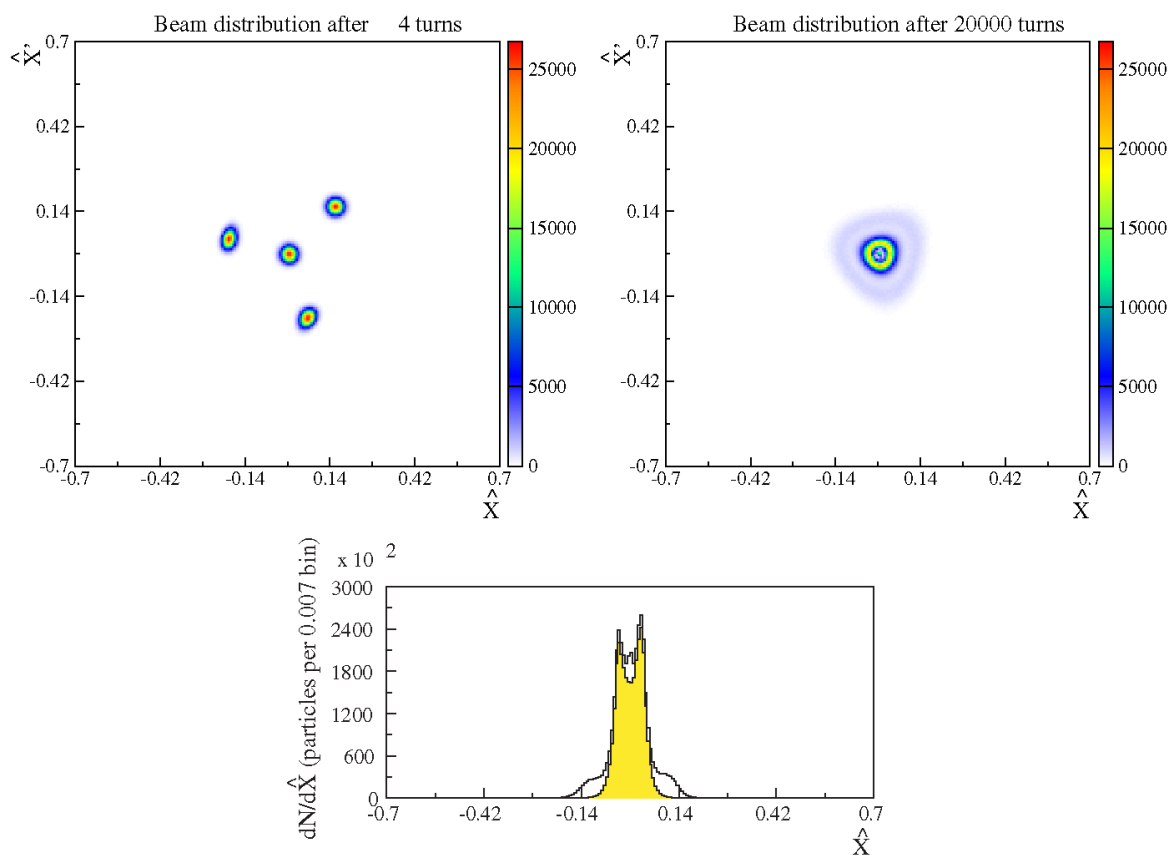

FIG. 6. (Color) Final distribution function for a variant of the three-turn injection with a fourth injected turn. The distribution at the end of the injection process is shown in the upper left part, while the final distribution is shown on the upper right part. The projection of the final beam distribution is plotted in the lower part. The extended halo is visible in both the final 2D beam distribution as well as its projection along the $\hat{X}$ axis. the mean value of the beam distribution. This approach is equivalent to fit an ellipse to the phase space distribution, where the fit parameters are its surface, amplitude, and orientation.

If the nominal Twiss parameters $\alpha, \beta$, $\gamma$, i.e., those relative to the nominal optics, are used to transform Eqs. (6) to normalized phase space via the well-known transformation rules [16]

$$
\hat{x}=\frac{x}{\sqrt{\beta}} \quad \hat{x}^{\prime}=\frac{\alpha}{\sqrt{\beta}} x+\sqrt{\beta} x^{\prime}
$$


the key relations

$$
\begin{gathered}
\left\langle\hat{x}^{2}\right\rangle=\frac{\beta^{*}}{\beta} \epsilon \quad\left\langle\hat{x} \hat{x}^{\prime}\right\rangle=\left(\alpha \frac{\beta^{*}}{\beta}-\alpha^{*}\right) \epsilon \\
\left\langle\hat{x}^{2}\right\rangle=\left(\alpha^{2} \frac{\beta^{*}}{\beta}-2 \alpha \alpha^{*}+\beta \gamma^{*}\right) \epsilon
\end{gathered}
$$

are obtained. The value of the rms emittance can be computed by solving Eqs. (8), namely,

$$
\epsilon=\left\langle\hat{x}^{2}\right\rangle\left\langle\hat{x}^{\prime 2}\right\rangle-\left\langle\hat{x} \hat{x}^{\prime}\right\rangle^{2} .
$$

The meaning of the coefficients $\beta^{*} / \beta$ and $\alpha \beta^{*} / \beta-\alpha^{*}$ is clear: they measure the deviation of the $1 \sigma$ contour of the beam distribution from the circular shape it should have in normalized phase space. In case of perfect matching, one would have $\beta^{*} / \beta=1$ and $\alpha \beta^{*} / \beta-\alpha^{*}=0$, implying the trivial relation $\beta^{*}=\beta, \alpha^{*}=\alpha$. Of course, Eqs. (8) should be transformed in the special coordinate system $\left(\hat{X}, \hat{X}^{\prime}\right)$ where the expression of the mismatch parameters will be unaffected, but the emittance will be transformed according to $\epsilon \rightarrow \epsilon / \lambda^{2}$.

In addition to the three parameters introduced so far, it is possible to introduce a fourth quantity, which was proposed recently as an indicator of the presence of beam halo [24], namely,

$$
h=\frac{\left\langle\hat{X}^{4}\right\rangle}{\left\langle\hat{X}^{2}\right\rangle^{2}}-2 .
$$

The quantity $h$ is the kurtosis of the beam distribution, which measures how the distribution is peaked with respect to a Gaussian. Following Ref. [24], the standard normalization is modified so that $h=0$ for KapchinskijVladimirskij distribution [25], which is known to feature no halo, while $h=1$ for a Gaussian. In the case of a hollow beam with a spatial distribution like

$$
\rho_{\text {proj }}(\hat{X})=\frac{1}{\sqrt{2 \pi} \sigma^{3}} x^{2} e^{-\left[x^{2} /\left(2 \sigma^{2}\right)\right]},
$$

then $h=-1 / 3$.

A series of numerical simulations were performed to determine the dependence of the parameters $\alpha \beta^{*} / \beta-\alpha^{*}$, $\beta^{*} / \beta, \epsilon_{\mathrm{final}}$, and $h$ at the end of the injection and merging processes as a function of the emittance for the injected beam $\epsilon_{\text {initial}}$, the slope of the tune curve during the resonance-crossing stage (assuming a linear tune variation), and the exponent $p$ of the tune curve during the resonance crossing. To test the robustness of the proposed injection method, the incoming beam was displaced horizontally with respect to the nominal center of the rightmost island and the whole injection process was studied under these special conditions. Both the four- and the three-turn injection schemes were considered. In the four-turn case (respectively three-turn one), the rightmost island is located at $\hat{X}=0.2209$ (respectively 0.1342 ) and the beam is displaced by $\Delta \hat{X}=0.0176$ (respectively 0.0192 ). The re- sults are shown in Figs. 7-9. Each injected turn is Gaussian-distributed in both $\hat{X}$ and $\hat{X}^{\prime}$. The number of initial conditions as well as the tune values $\left(\nu_{a}, \nu_{b}, \nu_{c}\right)$ are reported in the figures' captions.

It is worth stressing that additional emittance blowup might occur at the end of the whole process, when the beamlets have been merged into a single beam. In fact, in case the final equivalent optical parameters do not match the theoretical ones, filamentation could lead to an emittance given by [23]

$$
\begin{aligned}
\epsilon_{\text {after fil. }} & =H \epsilon_{\text {final }}, \\
\text { where } H & =\frac{1}{2}\left[\frac{\beta^{*}}{\beta}+\frac{\beta}{\beta^{*}}+\left(\alpha \sqrt{\frac{\beta^{*}}{\beta}}-\bar{\alpha} \sqrt{\frac{\beta}{\beta^{*}}}\right)^{2}\right] .
\end{aligned}
$$

As far as the dependence on the value of $\epsilon_{\text {initial }}$ is concerned (see Fig. 7), the mismatch parameters are in all cases considered almost equal to the perfect matched values of 0 and 1 , respectively. It is clearly seen that $\epsilon_{\text {final }}$ depends more critically on the position of the injected beam. This resembles the case of a standard injection, where a displacement of the injected beam generates beam filamentation and, eventually, emittance growth.

The fact that $\boldsymbol{\epsilon}_{\text {final }}$ tends to a finite value for vanishing $\epsilon_{\text {initial }}$ is due to the hollow shape of the final beam distribution. This observation is confirmed by the results for the parameter $h$, which tends to the value of -0.5 when $\epsilon_{\text {initial }} \rightarrow 0$. Indeed, this is the expected value for a projected distribution function of the type

$$
\rho_{\text {proj }}(\hat{X})=\frac{1}{a \pi \sqrt{1-\left(\frac{\hat{X}}{a}\right)^{2}}}
$$

resulting from a phase space distribution $\rho\left(\hat{X}, \hat{X}^{\prime}\right)=$ $\delta\left(\sqrt{\hat{X}^{2}+\hat{X}^{\prime 2}}-a\right)$, with $\delta(x)$ being the Dirac deltadistribution function. The distributions $\rho$ and $\rho_{\text {proj }}$ represent a very good approximation of the shape of the final beam, i.e., after complete filamentation, which is generated by an initial Gaussian distribution with vanishing value of the sigma.

As far as the dependence on the value of the slope of the tune curve is concerned (see Fig. 8), in general the four observables under study feature a very weak dependence on it. As for the previous case the mismatch parameters are essentially equal to the perfect-match case. For the fourthorder resonance, the value of $\epsilon_{\text {final }}$ reduces when the slope is smaller, probably due to the fact the resonance crossing is more adiabatic. Also in this case, the displaced injected beam features a poorer performance in terms of $\epsilon_{\text {final }}$.

Finally, the exponent of the tune curve seems to have almost no impact on the properties of the final beam after crossing the resonance.

From the results of the numerical simulations it is clear that the expected value of the emittance blowup after 

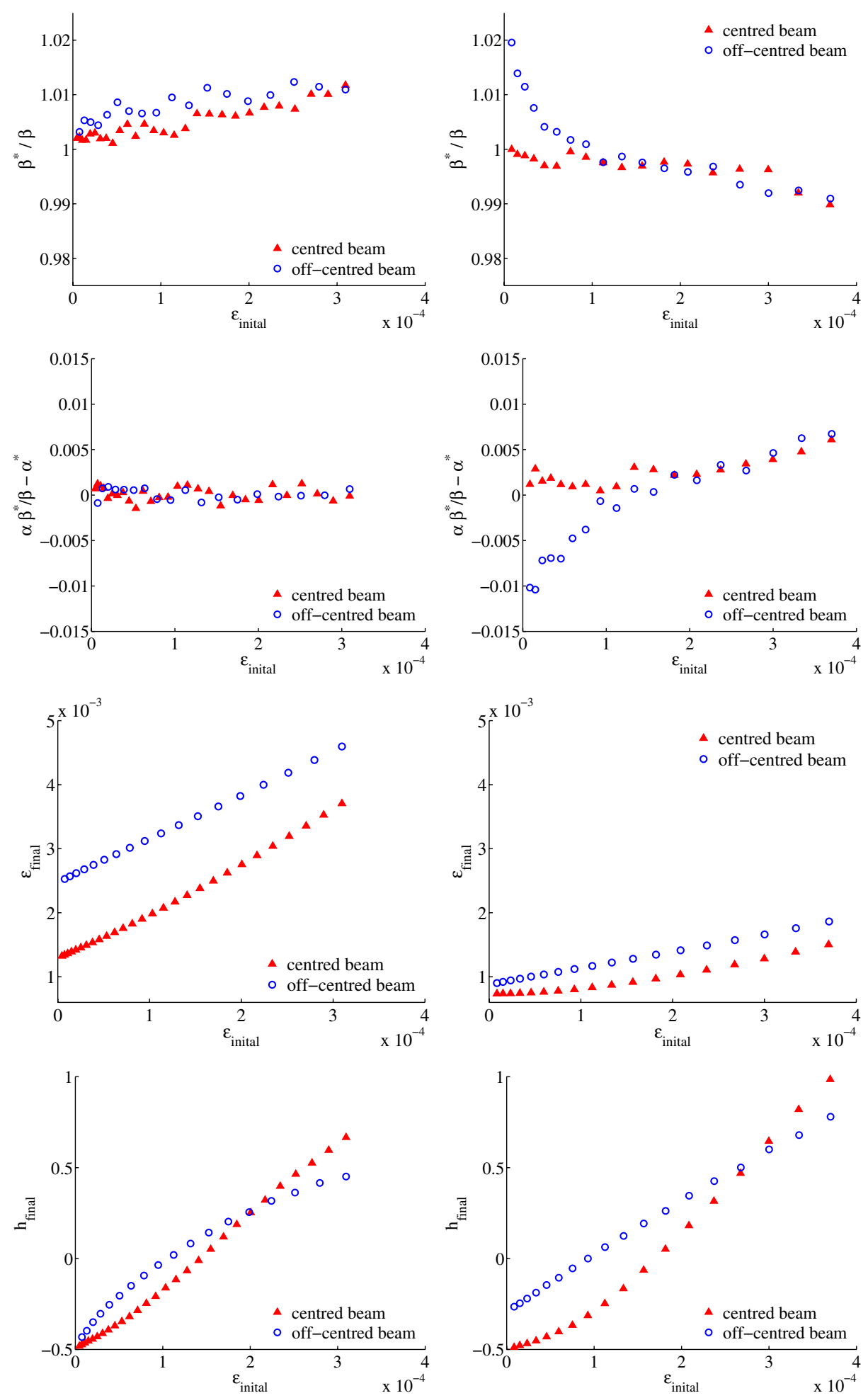

FIG. 7. (Color) Optical mismatch parameters, final emittance, and halo parameter as a function of the initial emittance of the injected beam for the four-turn injection (left column) and for the three-turn injection (right column). The solid triangles correspond to injecting the beam at the center of the rightmost island, while the open circles to injecting the beam displaced from the island's center. For the four-turn injection (respectively three-turn) the tune values are $\left(\nu_{a}, \nu_{b}, \nu_{c}\right)=(0.255,0.251,0.248)$ [respectively $\left.(0.328,0.341,0.35)\right]$, the number of initial conditions for each injected turn is $2.5 \times 10^{4}$ (respectively $10^{6}$ ), and the displacement of the off-centered beam is $\Delta \hat{X}=0.0176$ (respectively 0.0192 ). In both cases, the constant value of $p$ is 1 and $\left(n_{1}, n_{2}, n_{3}\right)=(12000,1,7999)$. 

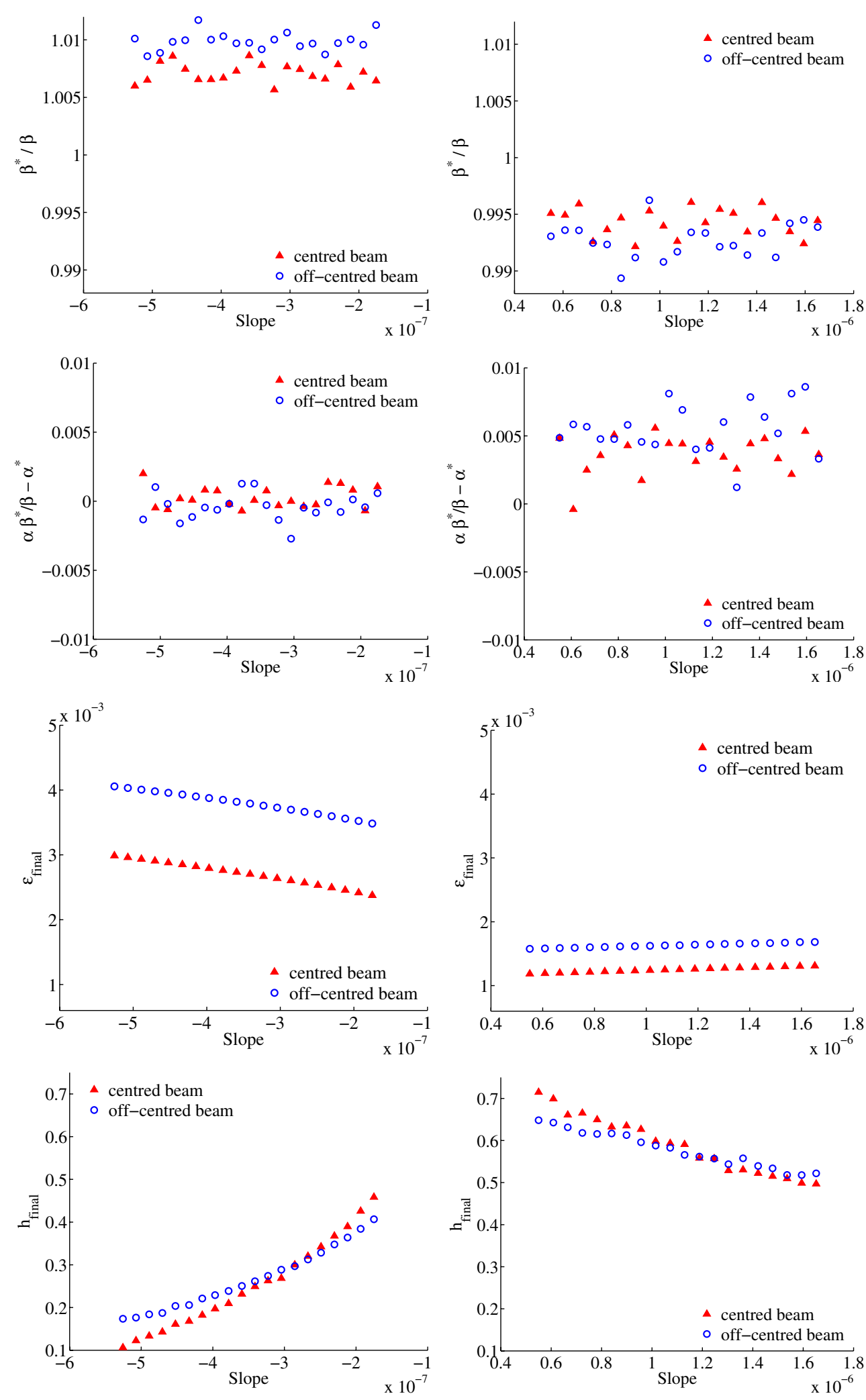

FIG. 8. (Color) Optical mismatch parameters, final emittance, and halo parameter as a function of the slope of the tune curve during the resonance crossing for the four-turn injection (left column) and for the three-turn injection (right column). The solid triangles correspond to injecting the beam at the center of the rightmost island, while the open circles to injecting the beam displaced from the island's center. For the four-turn injection (respectively three-turn) the tune values are $\left(\nu_{a}, \nu_{b}, \nu_{c}\right)=(0.255,0.251,0.248)$ [respectively $(0.328,0.341,0.35)]$, the constant value of $\epsilon_{\text {initial }}$ is 0.014 (respectively 0.017 ), and the displacement of the off-centered beam is $\Delta \hat{X}=$ 0.0176 (respectively 0.0192). In both cases, the number of initial conditions for each injected turn is $10^{6}$. 

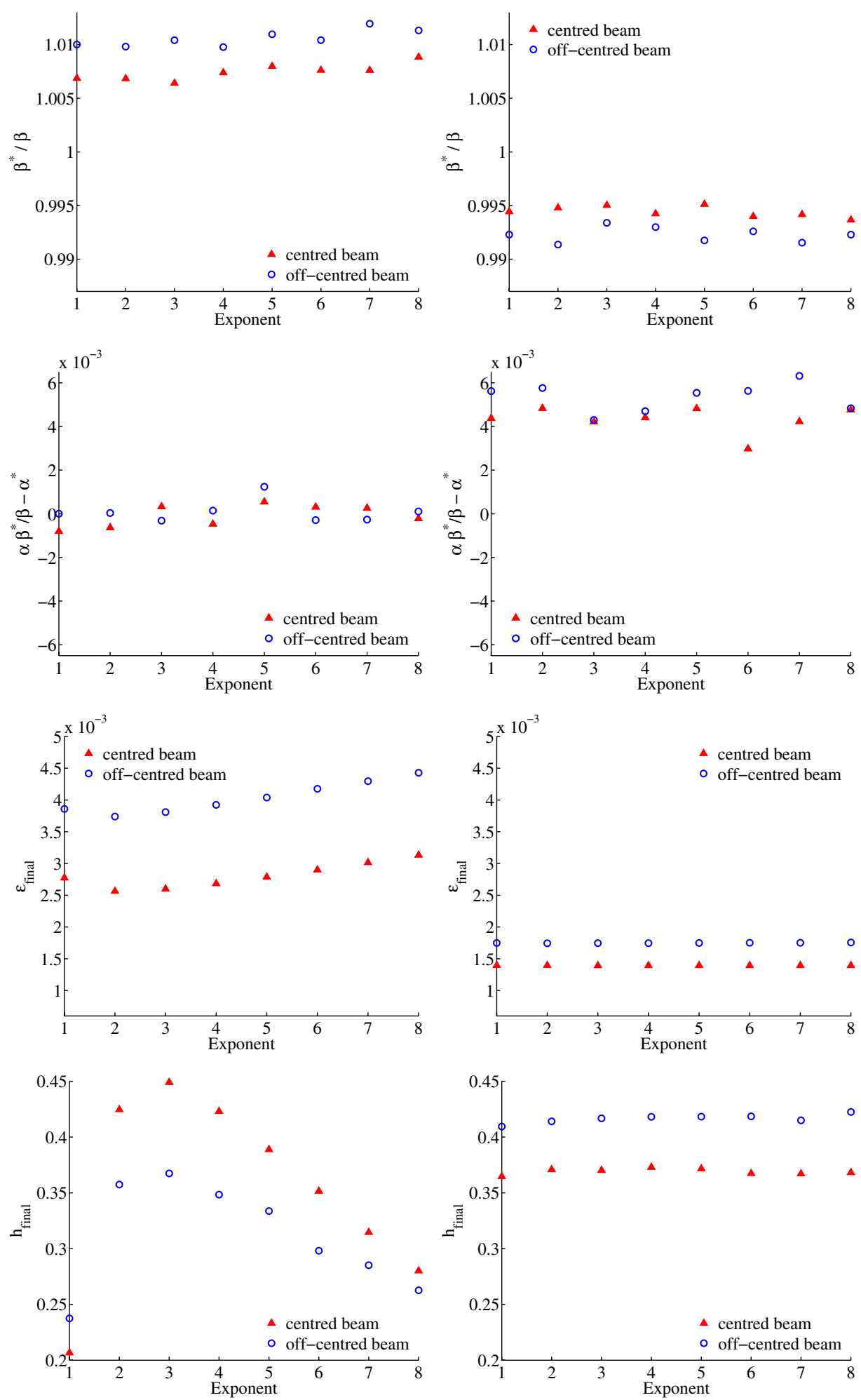

FIG. 9. (Color) Optical mismatch parameters, final emittance, and halo parameter as a function of the exponent $p$ of the tune curve for the four-turn injection (left column) and for the three-turn injection (right column). The solid triangles correspond to injecting the beam at the center of the rightmost island, while the open circles to injecting the beam displaced from the island's center. For the fourturn injection (respectively three-turn) the tune values are $\left(\nu_{a}, \nu_{b}, \nu_{c}\right)=(0.255,0.25,0.248)$ [respectively $\left.(0.328,0.333,0.35)\right]$, the constant value of $\epsilon_{\text {initial }}$ is 0.014 (respectively 0.017) and the displacement of the off-centered beam is $\Delta \hat{X}=0.0176$ (respectively $0.0192)$. In both cases, the constant value of $p$ is $1,\left(n_{1}, n_{2}, n_{3}\right)=(13000,500,6500)$ and the number of initial conditions for each injected turn is $10^{6}$. 
filamentation following the end of the merging process is almost negligible.

\section{COMPARISON BETWEEN NOVEL AND CLASSICAL MULTITURN INJECTION TECHNIQUES}

\section{A. A simple model for the classical multiturn injection}

For a complete performance analysis, it is important to compare the multiturn injection based on stable islands with the classical approach [1]. In this case, the closed orbit is modified so that the injected beam fills regions of the

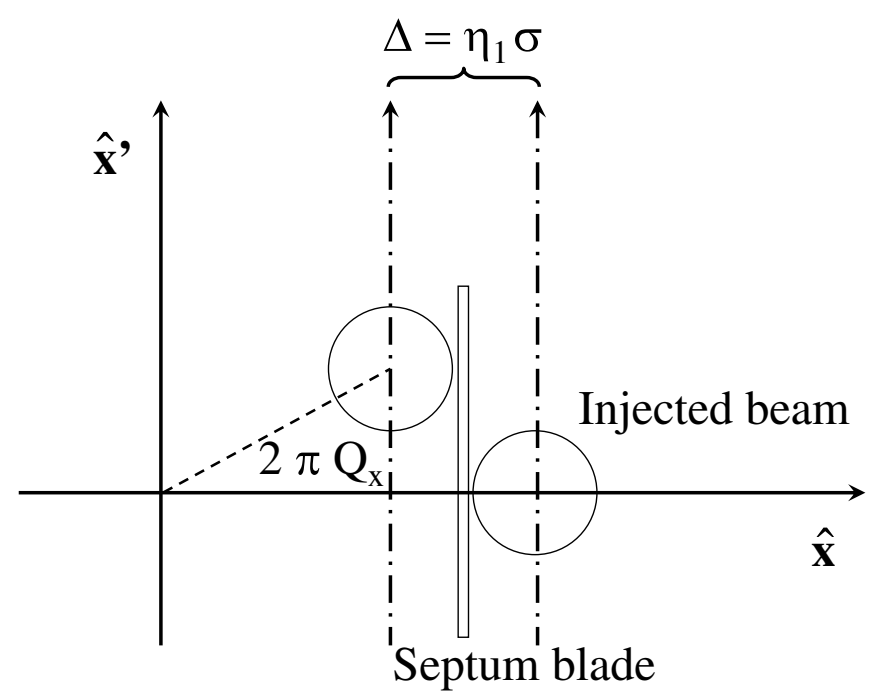

FIG. 10. Sketch of the injection model used to simulate the classical multiturn injection. Two types of reference systems are shown: the one represented by solid lines refers to the center of the vacuum chamber, i.e., the position of the nominal closed orbit once the injection bump is collapsed. The ones represented by dashed lines refer to the instantaneous closed orbit which moves together with the amplitude of the injection bump. transverse phase space, usually the horizontal one, at increasing amplitude. A sketch of the principle is shown in Fig. 10. The position of the closed orbit is initially located near the blade of the septum magnet. On a turn-by-turn basis the amplitude of the bump is collapsed and the closed orbit is moved towards the center of the vacuum chamber. By assuming that the average position of the beam injected at turn $l$ with respect to the closed orbit is given by

$$
\mu_{l}^{i}=\eta_{l} \sigma, \quad 0 \leq l \leq N-1,
$$

where $\sigma$ is the width of the beam distribution, assumed to be Gaussian, $\eta_{l}$ is the initial mean position of the $l$ th injected turn and $N$ is the total number of injected turns.

It is clear that the way the closed orbit is changed has a crucial impact on the properties of the beam after injection. For this reason two models were considered, namely, with $\eta$ being a linear function of the turn number $l$ and a second one where the position of the injected beam takes into account the rotation in phase space of the previously injected beam in order to minimize the value of the closed orbit change, namely,

$$
\eta_{l}=\eta_{l-1} \cos 2 \pi Q_{x}+\eta_{0}
$$

where $Q_{x}$ is the linear tune. The two functional forms can be written in a compact form as

$$
\eta_{l}=\eta_{0} \bar{\eta}_{l}=\left\{\begin{array}{l}
\eta_{0} l \\
\eta_{0} \frac{1-\cos ^{l} 2 \pi Q_{x}}{1-\cos 2 \pi Q_{x}} .
\end{array}\right.
$$

Strictly speaking, the latter form is valid when the fractional part of the tune $Q_{x}$ is smaller than 0.25 . It is also clear that the second model tends to the linear function in the limit $Q_{x} \rightarrow 0$. Also, it is readily found that in the latter case the position of the injected beam tends to a finite limit given by $\eta_{\infty}=\eta_{0} /\left(1-\cos 2 \pi Q_{x}\right)$. The different behavior of the injected beam position as a function of turn number is shown in Fig. 11. The effectiveness of the second scheme
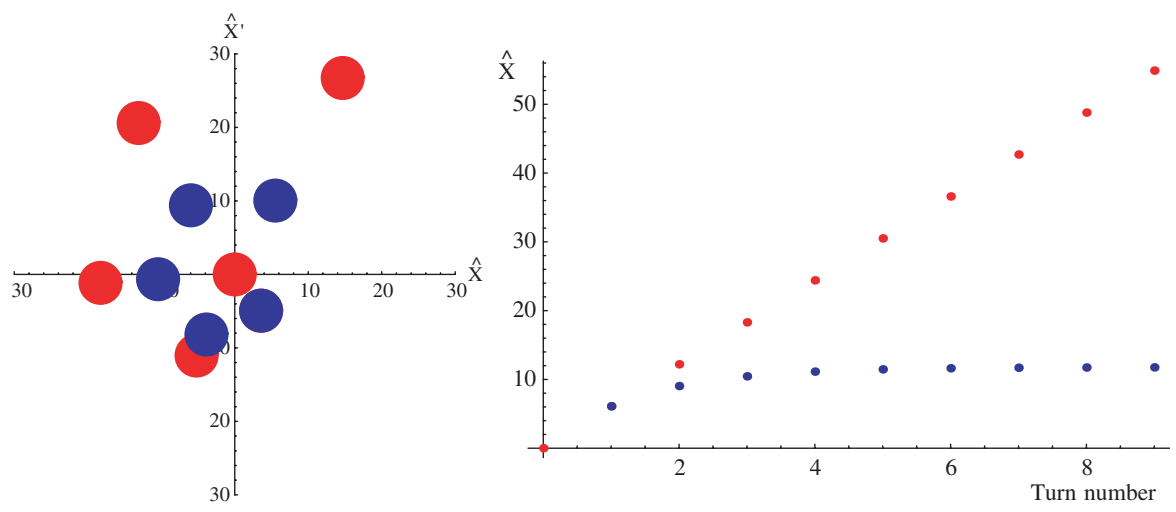

FIG. 11. (Color) Differences between the two models considered for the classical multiturn injection. The position of the injected beams is shown on the left panel (the disk radius represents the three-sigma level of the Gaussian distribution), while the amplitude vs the injected turn is plotted in the right panel. The linear increase of the first model is clearly shown (red markers), as well as the bounded behavior of the second one (blue markers). In all of the cases, the fractional part of the tune is 0.17 , while $\eta_{0}=6.1$. 
in distributing the injected beam in a smaller region around the origin of phase space is clearly visible.

The parameter $\eta_{0}$ is linked with the injection losses, as the amount of beam intercepted by the septum blade can be estimated as

$$
L\left(\eta_{0}\right)=2 \int_{\eta_{0}}^{\infty} G(\hat{X}, 0,1) d \hat{X}
$$

Therefore, its value has to be determined as a trade-off between losses minimization (requiring a large value of $\eta_{0}$ ) and minimization of the final emittance (requiring a small value of $\eta_{0}$ ). In all the subsequent computations the value of $\eta_{0}=6.1$, giving negligible injection losses, was used. This choice is imposed by the requirement of performing a fair comparison with the novel multiturn injection for which the losses, according to the results of the numerical simulations, are exactly zero.

The average beam position and angle at the end of the injection process are obtained as

$$
\mu_{l}^{f}+i \mu_{l}^{\prime f}=e^{2 \pi Q_{x} i(N-l)} \mu_{l}^{i}
$$

where the complex notation is used and $\mu^{\prime}$ stands for the average angle. The final beam distribution, which will be used to compute the emittance and the optical mismatch parameters using Eqs. (6), can be expressed as

$$
\rho^{f}\left(\hat{X}, \hat{X}^{\prime}\right)=\frac{1}{N} \sum_{j=0}^{N-1} \mathcal{G}\left(\hat{X}, \mu_{j}^{f}, \sigma\right) \mathcal{G}\left(\hat{X}^{\prime}, \mu_{j}^{\prime f}, \sigma\right),
$$

and $G(x, \mu, \sigma)$ represents a Gaussian distribution function with mean $\mu$ and width $\sigma$.

It is readily seen that the expressions for the mean values of the coordinates are given by

$$
\langle\hat{X}\rangle=\frac{1}{N} \sum_{j=0}^{N-1} \mu_{j}^{f} \quad\left\langle\hat{X}^{\prime}\right\rangle=\frac{1}{N} \sum_{j=0}^{N-1} \mu_{j}^{\prime f} .
$$

Similarly, expressions for the second-order moments can be obtained and are reported in Appendix A together with the corresponding formulas for the emittance and the optical mismatch parameters. There, the dependence on the free parameters, such as the number of injected turns and the position of the injected beam as a function of the turn number, is derived. As a first observation, the value of the final emittance depends linearly on that of the initial one. The slope of the linear relation depends on $\eta_{0}$ in the form of a fourth-order polynomial. The dependence on the tune and on the model used to change the position of the injected beam is too involved to be derived analytically.

The mismatch parameters are independent on the initial value of the emittance, and they are linked only to the details of the injection dynamics, i.e., how the injected beam position is varied, and on the linear tune. In this case the mismatch parameters are rational functions of polynomials in $\eta_{0}$ of degree two (numerator) and four (denominator).

\section{B. Comparison of final beam parameters}

Using the models presented in the previous section, a comparison of the performance of the classical injection with the novel method was carried out by means of numerical simulations. The quality factors used to qualify the two approaches are the optical mismatch parameters, namely $\alpha \beta^{*} / \beta-\alpha^{*}, \beta^{*} / \beta$, and $\epsilon_{\text {final }}$. Their dependence on the value of the emittance of the incoming beam is shown in Fig. 12. As already mentioned, $\eta_{0}=6.1$ and the linear model for $\eta_{l}$ was used. Four values of the linear tune were used for the simulation of the classical multiturn extraction. These results are compared with those for the proposed multiturn injection based on the fourth- (left part of Fig. 12) and third-order (right part of Fig. 12) resonances. It is important to stress that, in the case of the classical multiturn injection, the quality factors have been evaluated after a number of turns equal to that of novel multiturn injection to be compared with.

In agreement with the results of the analytical computations presented in Appendix A, the optical mismatch parameters for the classical injection scheme do not depend on $\epsilon_{\text {initial }}$, but only on $Q_{x}$ and such a variation is clearly non-negligible. The novel multiturn injection features values almost independent on the initial emittance and nearer to those corresponding to a perfect matching. Sensitivity to the number of injected turns, or on the resonance order, is also observed.

The evolution of the final emittance is also a crucial figure of merit. In fact, a smaller final emittance, hence smaller blowup with respect to the value of the injected beam emittance, implies less mechanical aperture required in the machine or, alternatively, a smaller amount of beam losses at injection. From Fig. 12 it is clear that $\epsilon_{\text {final }}$ behaves as predicted by the analytical estimates reported in Appendix A. The linear dependence of the final emittance from the initial one is also a feature of the novel multiturn injection process. Furthermore, provided the emittance of the incoming beam is not too small, the proposed injection is superior to the classical one in the sense that it generates smaller final emittance. Of course, for $\epsilon_{\text {initial }} \rightarrow 0, \epsilon_{\text {final }}$ tends to a finite value in the case of the novel multiturn injection, while $\epsilon_{\text {final }} \rightarrow 0$ for the classical multiturn injection. Hence, for rather small values of $\epsilon_{\text {initial }}$ the classical injection is performing better. These observations hold true, at least qualitatively, for both the four- and three-turn injection. The use of the linear dependence on $l$ of $\eta_{l}$ makes the comparison between the classical and the novel injection rather in favor of the latter as far as the final emittance is concerned. The use of the second model for $\eta_{l}$ improves the situation, but the injection based on stable resonances is still superior. The underlying principle is 

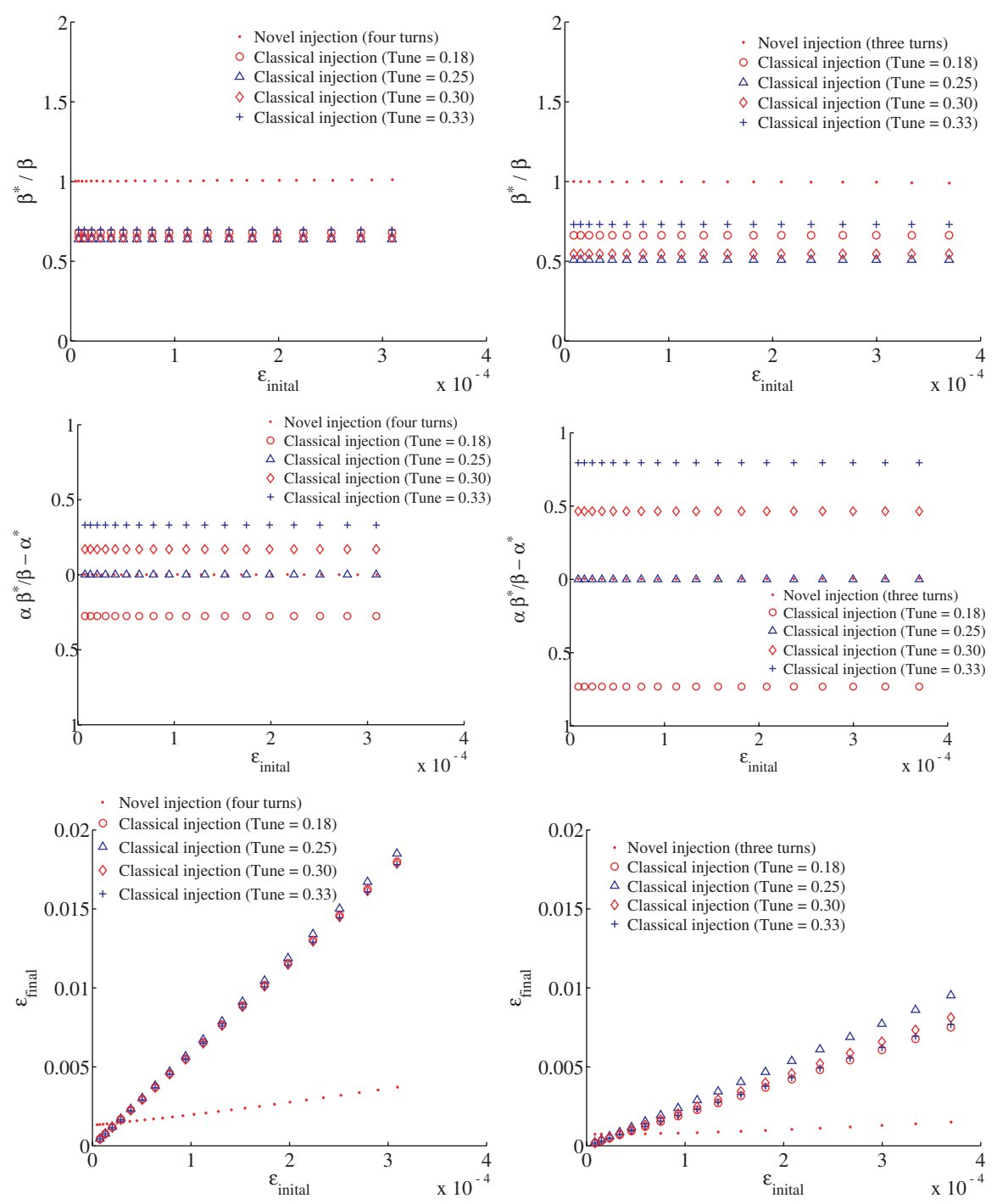

FIG. 12. (Color) Optical mismatch parameters and final emittance as a function of the initial emittance of the injected beam for simple model of the classical multiturn injection (markers) and the proposed multiturn injection based on the fourth-order resonance (left) and the third-order one (right). Four values of the linear tune have been considered for the classical multiturn injection.

responsible for this behavior. In fact, even though the beamlets are injected at rather large amplitudes, the resonance crossing allows moving them towards the center of the phase space thus reducing the value of the final emittance. In this respect the key ingredients are the trapping in the stable islands, to ensure that whenever the islands are moved the beam inside will follow, and the tune variation to displace the islands. It is clear that these elements are completely missing in the classical injection, thus making impossible to manipulate the injected beam emittance efficiently.

Finally, it is worth stressing that the beam will undergo filamentation once the injection process is over. In this sense the already larger final emittance of the classical injection will blow up even more due to the fact that the mismatch parameters are larger (see previous comments) and hence the factor $H$ will be larger.

\section{CONCLUSIONS}

In this paper a novel method to perform multiturn injection is presented. Such a technique is the time-reversal equivalent of the multiturn extraction recently proposed. The approach is based on the use of stable islands of transverse phase space generated by nonlinear magnetic element such as sextupoles and octupoles.

The proposed approach proved to work well according to the results of numerical simulations performed on a simple model describing the horizontal betatronic motion in a FODO cell with sextupoles and octupoles. No particle losses are observed in the cases studied and based on the use of either the fourth- or the third-order resonance. An interesting feature of the proposed method is that it allows generating hollow distributions in the transverse phase space. This feature could be extremely interesting in the 
case of multiturn injection of high-intensity, space-chargedominated beams.

A detailed analysis of the performance of the novel method entailed the construction of a simple model of the classical multiturn injection. For such a model, figures of merit like optical mismatch parameters and final value of the beam emittance, were computed analytically together with their dependence on other free parameters. A comparison between the two injection methods showed that the novel one is superior not only in terms of optical mismatch parameters, but also in the emittance blowup during injection defined as the ratio between the final and the initial, i.e., injected, beam emittance.

The next stage will be the detailed study of whether the peculiar transverse beam distribution could be conserved whenever space-charge effects are included in the numerical simulations and, in case, whether such a beam shaping is indeed beneficial for space charge. A good benchmark case could be the study of a $\mathrm{H}^{-}$injection based on the proposed approach.

\section{ACKNOWLEDGMENTS}

We would like to express our gratitude to S. Gilardoni and E. Métral for many interesting and stimulating discussions on this topic.

\section{APPENDIX: ANALYTICAL COMPUTATION OF FINAL BEAM PARAMETERS FOR CLASSICAL MULTITURN INJECTION}

The computation of the second-order moments of the final beam distribution (A1) can be obtained rather straightforwardly by applying the definition, namely,

$$
\begin{aligned}
\left\langle\hat{X}^{2}\right\rangle-\langle\hat{X}\rangle^{2} & =\sigma^{2}+\frac{1}{N} \sum_{k, l=0}^{N-1} \mu_{k} \mu_{l}\left(\delta_{k, l}-\frac{1}{N}\right) \\
\left\langle\hat{X} \hat{X}^{\prime}\right\rangle-\langle\hat{X}\rangle\left\langle\hat{X}^{\prime}\right\rangle & =\frac{1}{N} \sum_{k, l=0}^{N-1} \mu_{k} \mu_{l}^{\prime}\left(\delta_{k, l}-\frac{1}{N}\right) \\
\left\langle\hat{X}^{\prime 2}\right\rangle-\left\langle\hat{X}^{\prime}\right\rangle^{2} & =\sigma^{2}+\frac{1}{N} \sum_{k, l=0}^{N-1} \mu_{k}^{\prime} \mu_{l}^{\prime}\left(\delta_{k, l}-\frac{1}{N}\right),
\end{aligned}
$$

where the superscript $f$ was dropped from the average values and the phase space coordinates, and $\delta_{k, l}$ is the Kronecker delta defined as

$$
\delta_{k, l}= \begin{cases}0 & \text { if } k \neq l \\ 1 & \text { if } k=l\end{cases}
$$

Observing that $\epsilon_{\text {initial }}=\sigma^{2}$, then by applying Eqs. (6) one obtains

$$
\begin{aligned}
\left(\frac{\epsilon_{\text {final }}}{\epsilon_{\text {initial }}}\right)^{2}= & +\frac{1}{N} \sum_{k, l=0}^{N-1}\left(\bar{\mu}_{k} \bar{\mu}_{l}+\bar{\mu}_{k}^{\prime} \bar{\mu}_{l}^{\prime}\right)\left(\delta_{k, l}-\frac{1}{N}\right) \\
& +\frac{1}{N^{2}} \sum_{k, l, i, j=0}^{N-1}\left(\bar{\mu}_{k} \bar{\mu}_{l}+\bar{\mu}_{k}^{\prime} \bar{\mu}_{l}^{\prime}\right)\left(\delta_{k, l}-\frac{1}{N}\right) \\
& \times\left(\delta_{i, j}-\frac{1}{N}\right) \\
\alpha & \frac{\beta^{*}}{\beta}-\alpha^{*}=\frac{1}{N} \frac{\sum_{k, l=0}^{N-1} \bar{\mu}_{k} \bar{\mu}_{l}^{\prime}\left(\delta_{k, l}-\frac{1}{N}\right)}{\varepsilon} \\
\frac{\beta^{*}}{\beta} & =\frac{1+\frac{1}{N} \sum_{k, l=0}^{N-1} \bar{\mu}_{k}^{\prime} \bar{\mu}_{l}^{\prime}\left(\delta_{k, l}-\frac{1}{N}\right)}{\varepsilon},
\end{aligned}
$$

where $\varepsilon$ stands for the right-hand side of Eq. (A3) and the relation $\mu_{k}=\eta_{0} \bar{\mu}_{k}$ and similarly for the $\mu$ 's was used. These results show that $\epsilon_{\text {final }}$ depends linearly on the initial value of the emittance and that the mismatch parameters are independent on $\epsilon_{\text {initial }}$.

The previous equations can be recast in a different form to make explicit the dependence on the key parameter $\eta_{0}$, namely,

$$
\begin{aligned}
&\left(\frac{\epsilon_{\text {final }}}{\epsilon_{\text {initial }}}\right)^{2}=+\frac{\eta_{0}^{2}}{N} \sum_{k, l=0}^{N-1}\left(\bar{\mu}_{k} \bar{\mu}_{l}+\bar{\mu}_{k}^{\prime} \bar{\mu}_{l}^{\prime}\right)\left(\delta_{k, l}-\frac{1}{N}\right) \\
&+\frac{\eta_{0}^{4}}{N^{2}} \sum_{k, l, i, j=0}^{N-1}\left(\bar{\mu}_{k} \bar{\mu}_{l}+\bar{\mu}_{k}^{\prime} \bar{\mu}_{l}^{\prime}\right)\left(\delta_{k, l}-\frac{1}{N}\right) \\
& \times\left(\delta_{i, j}-\frac{1}{N}\right) \\
& \alpha \frac{\beta^{*}}{\beta}-\alpha^{*}=\frac{\eta_{0}^{2}}{N} \frac{\sum_{k, l=0}^{N-1} \bar{\mu}_{k} \bar{\mu}_{l}^{\prime}\left(\delta_{k, l}-\frac{1}{N}\right)}{\varepsilon} \\
& \frac{\beta^{*}}{\beta}=\frac{1+\frac{\eta_{0}^{2}}{N} \sum_{k, l=0}^{N-1} \bar{\mu}_{k}^{\prime} \bar{\mu}_{l}^{\prime}\left(\delta_{k, l}-\frac{1}{N}\right)}{\varepsilon}
\end{aligned}
$$

[1] G. Rees, in Proceedings of the CERN Accelerator School (CERN 94-01, 1994, Vol. 2, p. 331).

[2] G. I. Budker and G.I. Dimov, in Proceedings of the International Conference on High-Energy Accelerators, Dubna, 1963, p. 1372 (unpublished).

[3] R. Cappi and M. Giovannozzi, Phys. Rev. Lett. 88, 104801 (2002).

[4] R. Cappi and M. Giovannozzi, in Proceedings of the Eighth European Particle Accelerator Conference, edited by T. Garvey et al. (Institute of Physics, London, 2002), p. 1250.

[5] R. Cappi and M. Giovannozzi, in Proceedings of the 2003 Particle Accelerator Conference, edited by J. Chew et al. (IEEE, Piscataway, NJ, 2003), p. 2910. 
[6] R. Cappi and M. Giovannozzi, Phys. Rev. ST Accel. Beams 7, 024001 (2004).

[7] R. Cappi, M. Giovannozzi, M. Martini, E. Métral, G. Métral, A.-S. Müller, and R. Steerenberg, in Ref. [5], p. 388.

[8] M. Giovannozzi, R. Cappi, S. Gilardoni, M. Martini, E. Métral, A.-S. Müller, A. Sakumi, and R. Steerenberg, in Proceedings of the Ninth European Particle Accelerator Conference, edited by C. Petit-Jean-Genaz (Institute of Physics, London, 2004), p. 173.

[9] R. Cappi, S. Gilardoni, M. Giovannozzi, M. Martini, E. Métral, A.-S. Múller, and R. Steerenberg, in Proceedings of the 2005 Particle Accelerator Conference, edited by C. Horak (IEEE, Piscataway, NJ, 2005), p. 117.

[10] S. Gilardoni, M. Giovannozzi, M. Martini, E. Métral, A.-S. Müller, P. Scaramuzzi, and R. Steerenberg, Phys. Rev. ST Accel. Beams 9, 104001 (2006).

[11] M.J. Barnes, O.E. Berrig, A. Beuret, J. Borburgh, P. Bourquin, R. Brown, J.-P. Burnet, F. Caspers, J.-M. Cravero, T. Dobers, T. Fowler, S. Gilardoni, M. Hourican, W. Kalbreier, T. Kroyer, F. di Maio, M. Martini, V. Mertens, E. Métral, K. D. Metzmacher, C. Rossi, J.-P. Royer, L. Sermeus, R. Steerenberg, G. Villiger, and T. Zickler, CERN Report No. 2006-011, 2006, edited by M. Giovannozzi.

[12] M. Giovannozzi, R. Cappi, S. Gilardoni, M. Martini, E. Métral, A.-S. Müller, P. Scaramuzzi, and R. Steerenberg, in High Intensity and High Brightness Hadron Beams: 33rd ICFA Advanced Beam Dynamics Workshop on High Intensity and High Brightness Hadron Beams, AIP Conference Proceedings No. 773, edited by I. Hofmann,
J.-M. Lagniel, and R. W. Hasse (AIP, Melville, NY, 2005), p. 296.

[13] M. Giovannozzi and J. Morel, in Ref. [9], p. 1377.

[14] T. K. Khoe and R.J. Lari, in Proceedings of the 8th International Conference on High-Energy Accelerators, edited by M. H. Blewett (CERN, Geneva, 1971), p. 98.

[15] A. Bazzani, G. Servizi, E. Todesco, and G. Turchetti, CERN Yellow Report No. 94-02, 1994.

[16] E. Courant and H. Snyder, Ann. Phys. (Berlin) 3, 1 (1958).

[17] P. Scaramuzzi, Diploma thesis, University of Milan, 2004.

[18] R. Garoby and S. Hancock, in Proceedings of the Fourth European Particle Accelerator Conference, edited by $\mathrm{C}$. Petit-Jean-Genaz and V. Suller (World Scientific, Singapore, 1994), p. 282.

[19] A. Blas, S. Hancock, M. Lindroos, and S. Koscielniak, in Proceedings of the Seventh European Particle Accelerator Conference, edited by W. A. Mitaroff et al. (Institute of Physics, London, 2000), p. 1528.

[20] C. Carli and M. Chanel, in Ref. [4], p. 233.

[21] P. J. Bryant and K. Johnsen, Circular Accelerators and Storage Rings (Cambridge University Press, New York, 1993).

[22] J. Buon, in Ref. [1], 1994, p. 89.

[23] R. Cappi and M. Giovannozzi, CERN divisional report CERN/PS 2002-083 (AE), 2002.

[24] C. K. Allen and T. P. Wangler, Phys. Rev. ST Accel. Beams 5, 124202 (2002).

[25] I. M. Kapchinskij and V. V. Vladimirskij, in Proceedings of International Conference on High Energy Accelerators, edited by L. Kowarski (CERN, Geneva, 1959), p. 274. 